\title{
Optically mapping the electronic structure of coupled quantum dots
}

\author{
M. SCHEIBNER*, M. YAKES, A. S. BRACKER, I. V. PONOMAREV, M. F. DOTY, C. S. HELLBERG, \\ L. J. WHITMAN, T. L. REINECKE AND D. GAMMON* \\ Naval Research Laboratory, Washington, DC 20375, USA \\ *e-mail: scheibner@bloch.nrl.navy.mil; gammon@nrl.navy.mil
}

In a network of quantum dots ${ }^{1}$ embedded in a semiconductor structure, no two are the same, and so their individual and collective properties must be measured after fabrication. Here, we demonstrate a 'level anti-crossing spectroscopy' (LACS) technique in which the ladder of orbital energy levels of one quantum dot is used to probe that of a nearby quantum dot. This optics-based technique can be applied in situ to a cluster of tunnel-coupled dots, in configurations similar to that predicted for new photonic or quantum information technologies ${ }^{2-5}$. Although the lowest energy levels of a quantum dot are arranged approximately in a shell structure ${ }^{6-10}$, asymmetries or intrinsic physics-such as spin-orbit coupling for holesmay alter level splittings significantly ${ }^{11}$. We use LACS on a diatomic molecule composed of vertically stacked InAs/GaAs quantum dots and obtain the excited-state level diagram of a hole with and without extra carriers. The observation of excited molecular orbitals, including $\sigma$ and $\pi$ bonding states, provides fresh opportunities in solid-state molecular physics. Combined with atomic-resolution microscopy and electronicstructure theory for typical dots, the LACS technique could also enable 'reverse engineering' of the level structure and the corresponding optical response ${ }^{12}$.

To begin, we recall the previously established spectroscopic features of quantum dot molecules (QDMs). We use the recombination of an electron-hole pair (that is, an exciton $\mathrm{X}^{0}$ ) as an indicator for the state of the hole. In these structures, the electron is localized in the bottom dot (B dot) over the entire electric field range ${ }^{13-16}$. Recombination with the hole within the same dot leads to an intense spectral line $\left(\begin{array}{ll}1 & 0 \\ 1 & 0\end{array}\right)$ in the photoluminescence spectrum (Fig. 1). As the electric field is scanned, this intradot transition goes through the first of a series of anticrossings at a field that we take as $F=0$. Here, there is a resonance with the lowest interdot transition, $\left(\begin{array}{ll}1 & 0 \\ 0 & 1\end{array}\right)$, in which the hole is in the top-dot (T-dot) ground state. The interdot transition energy exhibits a strong field dependence (Stark shift), caused by a change in the relative level alignment between the two dots with electric field. The strength of the shift is determined by the dot separation. Its slope $\left(\Delta E / \Delta F=0.955 \mathrm{meV} \mathrm{kV}^{-1} \mathrm{~cm}\right)$ provides a built-in calibration for the conversion between electric field $(\Delta F)$ and energy $(\Delta E)$. This first resonance arises from the coherent tunnelling of the hole between the ' $s$ shells' of the two dots ( $\mathrm{B} 0$ and $\mathrm{T} 0$ ), and the corresponding formation of a bonding and an antibonding molecular state. Such resonances between the ground states of two quantum dots have been studied intensely in recent publications ${ }^{13-23}$.

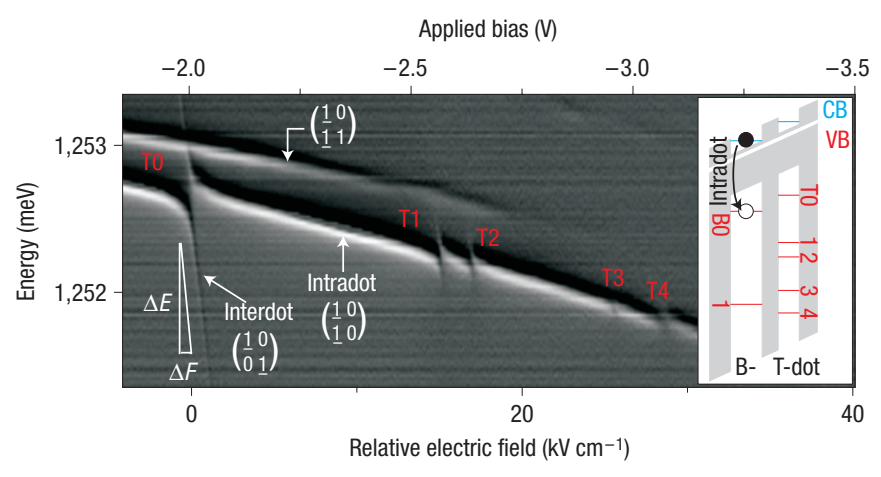

Figure 1 Principle of LACS. The electric-field-dependent optical spectrum of a neutral exciton in a quantum dot exhibits a sequence of anticrossings if a second quantum dot is placed next to it in the direction of the electric field. This sequence of anticrossings reveals the hole level structure of the T-dot ground state, T0, and excited states (T1-T4). The grey scale represents a (pixel) derivative with respect to the energy of the photoluminescence intensity. The slope of the interdot transition, $\Delta E / \Delta F$, can be used to convert between electric field and energy. $\left(\frac{e_{B} e_{T}}{h_{B}} h_{T}\right)$ gives the number of electrons and holes in the two dots and the underlines indicate the position of the recombining electron and hole. Right panel: Schematic diagram of the level structure of the coupled dot system for a field where the hole ground state in the $\mathrm{B}$ dot is between the T-dot ground state and the first excited state $\mathrm{T1}$.

As we increase the electric field from $F=0$, we find that the intradot exciton transition exhibits not only one but a whole sequence of distinct anticrossings. The extra anticrossings seem to be of the same nature as that of the lowest resonance. That is, they form where the intradot transition intersects with interdot transitions, which are optically weak away from the intersection. We intuitively identify these extra anticrossings with resonances between the ground hole state of the B dot (B0) and the excited hole states in the $\mathrm{T}$ dot (T1-T4 in Fig. 1). Consequently, the separation between the anticrossings corresponds to the spacing between hole levels in the $\mathrm{T}$ dot, and thus we have a direct and precise measurement of the hole energy-level structure of this $\mathrm{T}$ dot. For example, the spectrum shown in Fig. 1 seems to follow a shell structure with a fairly large energy gap between the $s$ level and the two $p$ levels (T1 \& T2), which are only slightly split. 

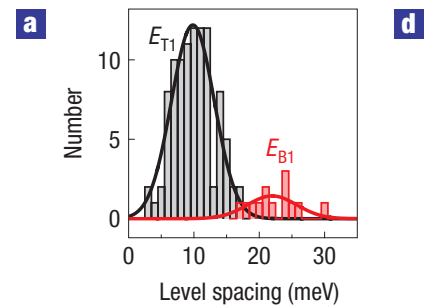

b
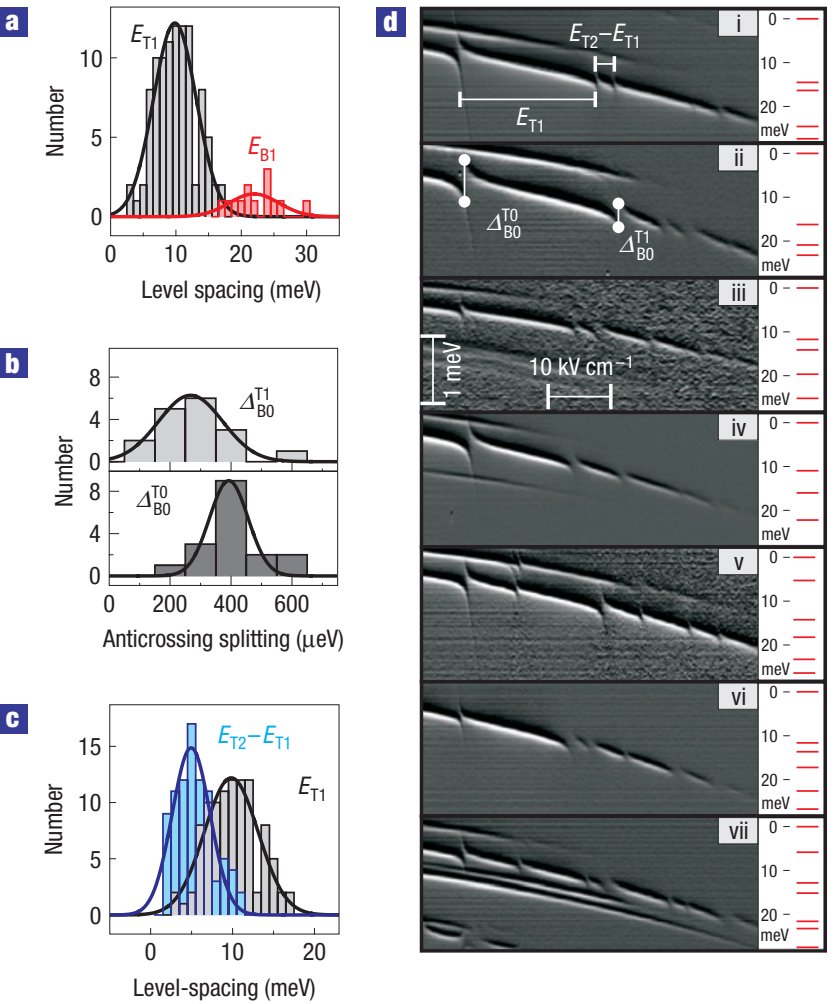

Figure 2 Statistics of the energy-level structure. a, The distribution of the splitting between the ground state and the first excited state for the T dot (grey, $E_{\mathrm{T} 1}$ ) and the $\mathrm{B}$ dot (red, $\left.E_{\mathrm{B} 1}\right)$. The solid lines are gaussian fits with average values of $9.8 \mathrm{meV}$ and $22.0 \mathrm{meV}$ and standard deviations of $3.3 \mathrm{meV}$ and $3.8 \mathrm{meV}$. $\mathbf{b}$, The anticrossing splitting for the resonance between the hole ground states of both dots (bottom, $\Delta_{\mathrm{BO}}^{\mathrm{TO}}$ ) and the resonance between the B-dot ground state and the first excited state in the $\mathrm{T}$ dot (top, $\left.\Delta_{\mathrm{B} 0}^{\mathrm{T}}\right)$ ). The solid lines are gaussian fits with average values of $393 \mu \mathrm{eV}$ and $267 \mu \mathrm{eV}$ and standard deviations of $63 \mu \mathrm{eV}$ and $105 \mu \mathrm{eV}$. c, The distributions of the level spacings between the ground state, $\mathrm{T0}$, and the first excited state, T1 (grey), and between the first and second excited state, T2 (blue). d, LACS examples of the neutral exciton and extracted T-dot hole level structure (see Supplementary Information, Table S2 for the values).

From measurements of a large number of examples (Fig. 2a), we find an average value for the energy splitting between the first two anticrossings of $\bar{E}_{\mathrm{T} 1}=10 \mathrm{meV}$. We will show below how to obtain the value for the splitting in the B dot; it is $\bar{E}_{\mathrm{B} 1}=22 \mathrm{meV}$ for the neutral exciton. The structure of the dots was measured with cross-sectional scanning tunnelling microscopy (XSTM) from another wafer with identically grown QDMs (but with density ten times higher). These results are shown in Fig. 3a. A well-known feature is observed in our molecules, with the strain-nucleated $\mathrm{T}$ $\operatorname{dot}(18 \mathrm{~nm}$, average width) wider than the B $\operatorname{dot}(14 \mathrm{~nm})$. Figure $3 \mathrm{~b}$ summarizes this result for many molecules. The large difference between $\bar{E}_{\mathrm{T} 1}$ and $\bar{E}_{\mathrm{B} 1}$ can then be explained qualitatively if these splittings are influenced significantly by the lateral size of the B and $\mathrm{T}$ dots in the molecules, with holes in the $\mathrm{B}$ dot confined more strongly $y^{24-26}$.

The magnitude of the anticrossing splittings between the bonding and antibonding states are also revealing. Figure $2 \mathrm{~b}$ shows the distribution of these anticrossing energies for the hole ground states (the ' $s$-shell' states of the two dots). The magnitude of this anticrossing energy is proportional to the wavefunction overlap of the hole states in the two dots, which depends strongly on both the barrier thickness ${ }^{14}$ and the symmetries of the wavefunctions.
We find that the corresponding anticrossing energies for the first excited state are on average smaller, as seen in Fig. 2b. This behaviour is consistent with the shell model, where the overlap integral of the ' $s$ shell' of the B dot with the ' $p$ shell' of the T dot should be small because of the symmetry. It is not zero because the symmetry is not perfect.

We present our results using the language of the shell model, but this must be viewed as an approximation. In many cases this correspondence is compelling as we note. Comparison with detailed theory, that accounts for spin-orbit interaction, strain and so ${ }^{11,27}$, will lead to a better understanding of the spectra, and perhaps to the spectral identification of model quantum dots (with minimal asymmetries). However, fluctuations in the spectra from dot to dot are substantial and also interesting. With level anticrossing spectroscopy (LACS) for many examples, level structure fluctuations become apparent (Fig. 2d). Not only does the spacing between the ground state and the first excited state vary from dot to dot (Fig. 2a), but also the splittings of the ' $p$ shell', that is, first excited state to second excited state (Fig. 2c), making the overall pattern of the spectrum look quite different in many cases. For example, although we find that the energy to the first excited state is often substantially larger than that between the first two excited states, this is not always true (see Fig. $2 \mathrm{~d}(\mathrm{v}$,vii)). Of course, shape asymmetries will lead to changes in the splittings, but there will also be other contributions, such as variations in the compositional profile of the dots and intrinsic effects such as spinorbit coupling.

The excited-state spectrum of the hole in the T dot from Fig. 1 is not the level structure of a bare hole because it was obtained in the presence of an electron in the B dot. As we now discuss, we have also measured the hole spectrum without the extra electron. Figure 4 shows LACS of a singly positively charged exciton $\left(\mathrm{X}^{+}\right)$. From previous work, it is known that the transitions of the $\mathrm{X}^{+}$map the level resonances of both the initial $\left(\mathrm{X}^{+}\right)$and final $(\mathrm{h})$ states. This causes an ' $\mathrm{x}$ ' pattern to be formed in the vicinity of the ground-state resonances of the bare hole $(\mathrm{h})$ and the $\mathrm{X}^{+}$(refs 13,15), as outlined in Fig. 4 by the yellow dashed rectangle. By following the intradot $\mathrm{X}^{+}$transition $\left(\frac{1}{2} 0^{0}\right)$ to the right towards higher fields, we find again a sequence of anticrossings, that is similar to the case of the $\mathrm{X}^{0}$, $\left(\begin{array}{l}1^{0} \\ 1_{0}\end{array}\right)$ (to the right of the orange rectangle in Fig. 4). However, for the charged exciton, pairs of anticrossings are observed for each excited state. In each case, an $\mathrm{X}^{+}$anticrossing is followed by a hole anticrossing at slightly higher fields. As an example, we have pinpointed the first pair of T-dot excited-state anticrossings with a yellow triangle for the $\mathrm{X}^{+}$anticrossing and a circle for the bare-hole anticrossing, both labelled with T1. The full excited-state ' $\mathrm{x}$ ' patterns are not observed because relaxation from the excited state occurs before luminescence can occur. To support the assignments, a model calculation of the level diagram and the corresponding $\mathrm{X}^{+}$ transitions has been fitted to the data and is shown in Fig. 5 .

The sequence of hole anticrossings provides a measure of the bare-hole excited-state spectrum of the $\mathrm{T}$ dot. Moreover, the sequence of $\mathrm{X}^{+}$anticrossings gives the hole spectrum in the presence of an $\mathrm{e}-\mathrm{h}$ pair in the $\mathrm{B}$ dot, as shown on the righthand side of Fig. 4. We can thus analyse how the excited-state splittings are perturbed by the presence of extra charges and find that the extra particles in the B dot have a small influence on the excited-state splittings of the $\mathrm{T}$ dot in this sample. For the three charge configurations, $\mathrm{X}^{0}, \mathrm{X}^{+}$and the bare hole, we measure a splitting between the T-dot ground state and the first excited state of $8.0 \mathrm{meV}, 6.8 \mathrm{meV}$ and $6.6 \mathrm{meV}$ respectively.

We have shown how to obtain the spectrum of the bare hole in the $\mathrm{T}$ dot. Similar information is obtained for the B dot. By following the $\mathrm{X}^{+}$intradot transition $\left(\begin{array}{c}1_{1}^{0} \\ 11\end{array}\right)$ to the left of the groundstate ' $x$ ' pattern in Fig. 4, more anticrossings are observed. The first 

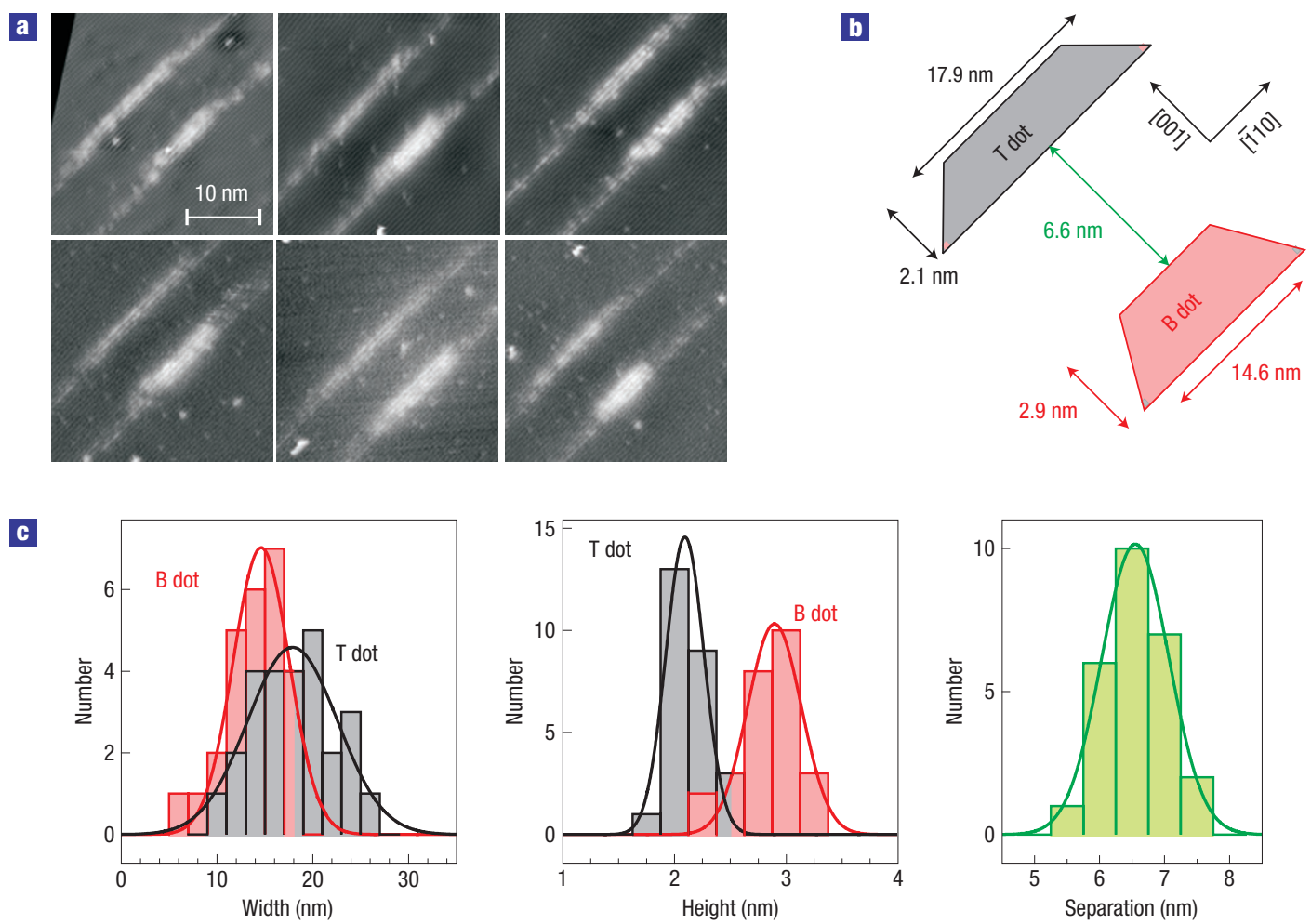

Figure 3 XSTM study of QDMs. a, XSTM images of QDMs similar to those studied with LACS. $\mathbf{b}$, Schematic diagram of a QDM. The values represent the average dimensions obtained from gaussian fits of the dot widths, dot heights and the dot separation shown in $\mathbf{c}$.

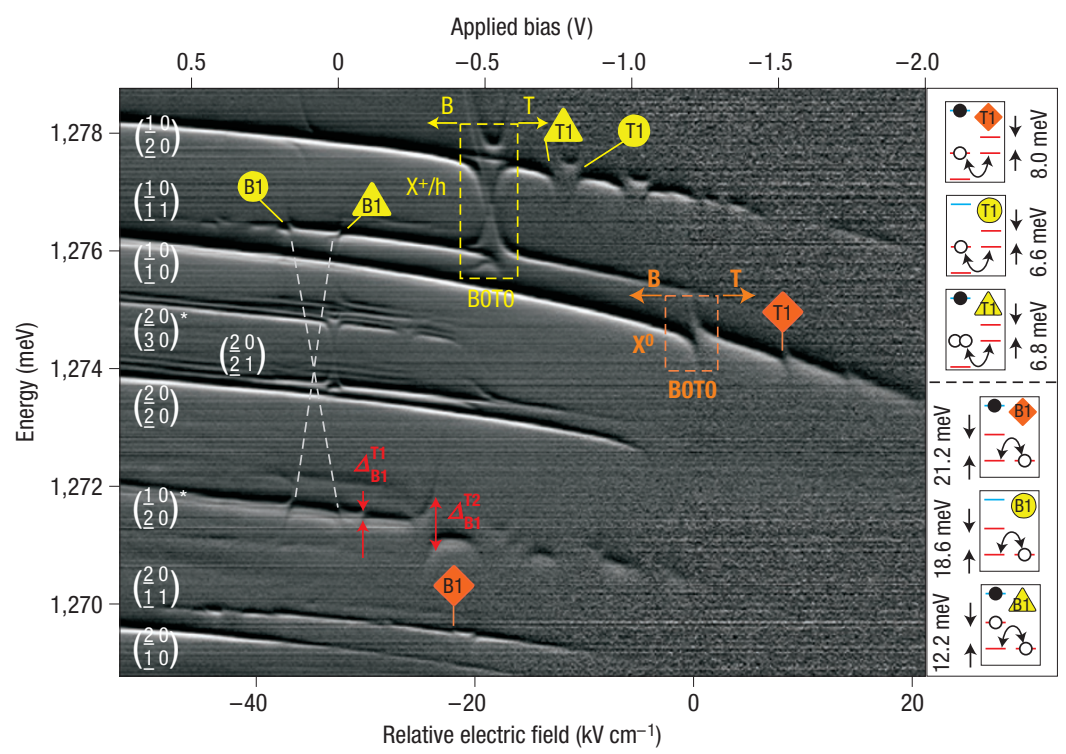

Figure 4 Versatility of LACS. The field-dependent photoluminescence spectrum of a QDM shows anticrossing sequences for several charge configurations. The dashed rectangles mark resonances between the hole ground states (BOT0) of both dots for the neutral exciton, $\mathrm{X}^{0}$ (orange rectangle), the positive trion, $\mathrm{X}^{+}$, and the bare hole, $\mathrm{h}$ (both in the yellow rectangle). For each charge configuration, the $\mathrm{T}$ (B)-dot hole level sequence is found to the right (left) of the corresponding rectangle. The grey dashed lines indicate an excited-stated ' $x$ ' pattern formed by the B1 resonances of $X^{+}$and $h$. Analogous behaviour is found for the $\left(\begin{array}{c}2 \\ 1 \\ 1\end{array}\right)$ transition, which maps the B-dot level structure if the system is occupied by an $\mathrm{X}^{0}$ or $\mathrm{XX}^{0}$. The right panel shows the correspondingly marked anticrossings and gives the values extracted for the energy spacing between the ground to first excited states of both dots. Further anticrossings are discussed in Supplementary Information, Fig. S1 and Table S1.

two are part of an excited-state ' $x$ ' pattern (grey dashed lines Fig. 4). The ' $\mathrm{x}$ ' pattern extends down in energy to the transition $\left(\begin{array}{c}1 \\ \frac{1}{2} \\ 2\end{array}\right)^{\circ}$ that arises from an excited triplet state, as shown in Fig. 5a. The corresponding singlet is not observed because of fast relaxation, 


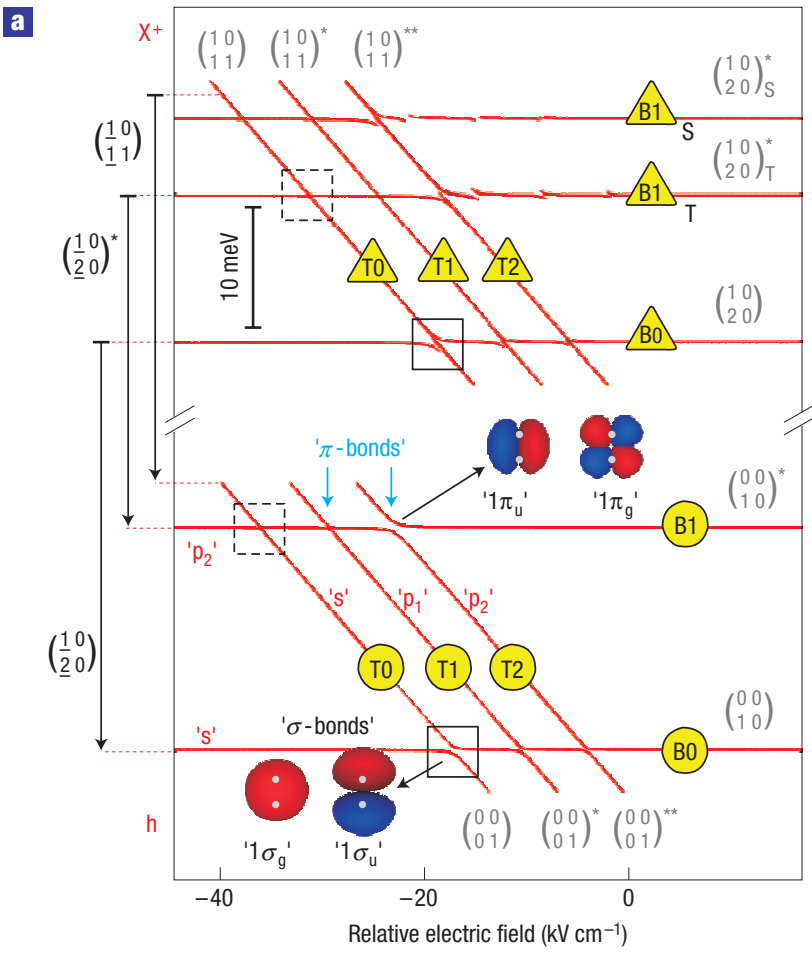

b

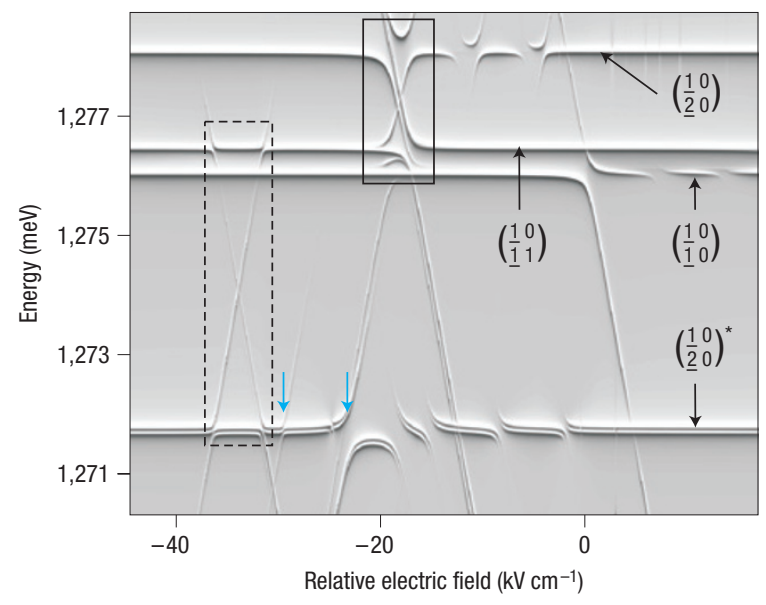

Figure 5 Calculated spectrum. a, Calculated $\mathrm{h}$ and $\mathrm{X}^{+}$energy levels of the QDM from Fig. 4. The calculations account for five hole levels (two in the $B$ dot and three in the T dot) and one electron level in the B dot. Note, for clarity, only the $X^{+}$levels that correspond to the five hole states but with an extra hole in the BO level and an extra electron are drawn. The $\left(\begin{array}{ll}1 & 0 \\ 2 & 0\end{array}\right)^{*}$ state is split into a singlet (S) and three (not resolved) triplet states $(\mathrm{T})$. For further details on the calculations, see the Supplementary Information. The schematic diagrams show analogue 'gerade' and 'ungerade' $1 \sigma$ - and $1 \pi$-bonds of real homonuclear diatomic molecules. $\mathbf{b}, \mathrm{A}$ fit of the $\mathrm{X}^{+}$transition spectrum in Fig. 4 derived by computing the optically allowed transitions from the $X^{+}$to the $h$ levels. The $X^{0}$ spectrum is also included here. The ' $X$ ' pattern in the dashed (solid) rectangle is a result of the two anticrossings in the dashed (solid) rectangles in a.

unlike the triplet in which case Pauli blocking prevents relaxation (see Supplementary Information, Fig. S1 for detailed assignments). From the analysis of this pattern, we obtain the level spacing between the ground state and the first excited hole state of the
$B$ dot (both the bare-hole energy and that in the presence of an extra e-h pair). The level spacings in the B dot are more strongly dependent on the charge configuration than those of the $\mathrm{T}$ dot (see right-hand side of Fig. 4) because in this case the charges are in the same dot. In particular, for the $\mathrm{X}^{+}$, in which the hole excitation energy is measured in the presence of an extra e-h pair, a strong $\mathrm{h}-\mathrm{h}$ exchange energy accounts for much of a significant reduction in ground to first excited energy spacing $(12.2 \mathrm{meV})$ compared with that of the bare hole $(18.6 \mathrm{meV})$. We note that level spacings have also been measured in the neutral and charged biexciton ${ }^{16}$ transitions with consistent results.

As a final and especially interesting example of solid-state molecular physics, we consider the resonances between the ' $p$-shell' states (Fig. 5a). The molecular orbitals formed at these anticrossings are the QDM analogue to $\pi$ bonds in real molecules, as opposed to the $\sigma$ bonds made up of the $s$ states. They are identified with the blue arrows in the calculated level diagram and spectrum in Fig. 5, and are measured in the excited $\mathrm{X}^{+}$line $\left.\left(\frac{1}{2}\right)^{0}\right)^{*}$ in Fig. 4. We expect from the shell model that the first two excited states of the $T$ dot (T1 and T2) correspond primarily to $\mathrm{p}_{1}$ and $\mathrm{p}_{2}$, and that the overlap integral with the $\mathrm{p}_{1}\left(\right.$ or $\left.\mathrm{p}_{2}\right)$ of the $\mathrm{B}$ dot (B1) will be large in one case and small in the other because of symmetry. In fact, this is exactly what we find in this example. In real molecules, one would be $\mathrm{p}_{x}$ and the other $\mathrm{p}_{y}$. The anticrossing energy between $\mathrm{B} 1$ and $\mathrm{T} 1$ is very small $\left(\Delta_{\mathrm{B} 1}^{\mathrm{T} 1}=100 \mu \mathrm{eV}\right)$ compared with that between the two $s$ states $\left(\Delta_{\mathrm{B} 0}^{\mathrm{T} 0}=610 \mu \mathrm{eV}\right)$, whereas that between $\mathrm{B} 1$ and T2 is large $\left(\Delta_{\mathrm{B} 1}^{\mathrm{T}}=870 \mu \mathrm{eV}\right)$. This is seen most clearly in the calculated level diagram in Fig. 5a. This result implies that B1 and T2 have the same symmetry, and that the energy ordering of the $p$-shell states is opposite in the two dots.

\section{METHODS}

The two samples measured with photoluminescence were grown by molecular beam epitaxy on top of an $\mathrm{n}$-GaAs substrate with the following layer sequence: $500 \mathrm{~nm} \mathrm{n}^{+}$-GaAs (buffer), $80 \mathrm{~nm}$ i-GaAs, coupled dot layer, $230 \mathrm{~nm}$ i-GaAs, $40 \mathrm{~nm} \mathrm{Al} \mathrm{A}_{0.3} \mathrm{Ga}_{0.7} \mathrm{As}, 10 \mathrm{~nm}$ i-GaAs, $8 \mathrm{~nm}$ titanium (semi-transparent). The coupled dot layer from Figs 1 and 2 (Fig. 4) consisted of a B dot with $2.5 \mathrm{~nm}$ $(3.0 \mathrm{~nm})$ nominal height and a $\mathrm{T}$ dot of $2.5 \mathrm{~nm}(2.3 \mathrm{~nm})$ nominal height separated by an i-GaAs tunnel barrier of $6 \mathrm{~nm}$. The height of the quantum dots, and thereby their ground-state transition energies, are controlled by an indium flush technique, where the dots are partially capped with GaAs and the exposed top part of the dots is removed before they are completely buried in GaAs (refs 28,29). These quantum dots are a few nanometres thick and $10-20 \mathrm{~nm}$ wide. To access individual pairs of quantum dots, 120 -nm-thick aluminium shadow masks with $1-\mu \mathrm{m}$-diameter apertures were put on the top surface of these $\mathrm{n}^{+}$Schottky diode structures. We chose dot pairs in which the B dot exhibited a lower exciton ground-state transition energy than the $\mathrm{T}$ dot. The samples were mounted on the coldfinger of a helium continuous flow cryostat and kept at a temperature of about $10 \mathrm{~K}$. The quantum dots were excited quasi-resonantly (below the wetting layer) with a frequency-tunable titanium-sapphire laser. The photoluminescence signal was spectrally dispersed with a $0.75 \mathrm{~m}$ monochromator equipped with a $1,200 \mathrm{~mm}^{-1}$ line grating and collected with a liquid-nitrogen-cooled CCD (charge-coupled device) camera. The overall spectral resolution of the set-up was $50 \mu \mathrm{eV}$. The grey scale plots were obtained by calculating the change of photoluminescence intensity with energy, defined as $[I(E, F)-I(E+\Delta E, F)] /[I(E, F)-I(E+\Delta E, F)]$, where $I(E, F)$ is the photoluminescence intensity at a certain energy, $E$, and field, $F$, and $\Delta E$ is an energy difference, which is given by the pixel separation on the CCD camera and the dispersion of the monochromator.

XSTM images were acquired as in ref. 30. Samples were scribed in situ and cleaved along the [110] direction. The original scans were nominally $512 \times 512$ pixels and $38.4 \times 38.4 \mathrm{~nm}$. All images were acquired using constant current $(0.06-0.12 \mathrm{nA})$ and filled electronic states $(2-3 \mathrm{~V})$. Images in Fig. 3a were processed to correct for thermal drift and line-to-line noise. In Fig. 3a, the bottom row contains $30.4 \mathrm{~nm} \times 30.4 \mathrm{~nm}$ images of the $3.0 \mathrm{~nm} \mathrm{~B} \mathrm{dot,} 6 \mathrm{~nm}$ barrier and $2.3 \mathrm{~nm} \mathrm{~T}$ dot capped at $520^{\circ} \mathrm{C}$. The images are taken at sample bias $-2 \mathrm{~V}$. The top row contains $30.4 \mathrm{~nm} \times 30.4 \mathrm{~nm}$ images of dots grown with a 
similar recipe except capped at $480^{\circ} \mathrm{C}$. The top right image is at $-2 \mathrm{~V}$ sample bias and the top left and middle images are at $-3 \mathrm{~V}$ sample bias.

The histograms in Fig. 3c were generated from a set of 26 images of coupled quantum dots taken with atomic resolution processed with only a plane subtraction. The sizes were determined by visually marking a four-sided boundary at the border of each quantum dot, then measuring the average width and height of each quantum dot region, using the GaAs lattice as a length calibration. Owing to the difficulty of determining the boundary visually, the error on any individual measurement is around $20 \%$, except for the spacing measurement, which is $10 \%$. In addition, as the quantum dots are not expected to be cleaved along the plane of greatest size, the measured value probably represents an underestimate of the true average size of the quantum dots.

\section{Received 7 August 2007; accepted 7 January 2008; published 10 February 2008.}

\section{References}

1. Stangl, J., Holý, V. \& Baur, G. Structural properties of self-organized semiconductor nanostructures. Rev. Mod. Phys. 76, 725-783 (2004)

2. Biolatti, E., Iotti, R. C., Zanardi, P. \& Rossi, F. Quantum information processing with semiconductor macroatoms. Phys. Rev. Lett. 85, 5647-5650 (2000).

3. Unold, T., Mueller, K., Lienau, C., Elsaesser, T. \& Wieck, A. D. Optical control of excitons in a pair of quantum dots coupled by the dipole-dipole interaction. Phys. Rev. Lett. 94, 137404 (2005).

4. Emary, C. \& Sham, L. J. Optically controlled logic gates for two spin qubits in vertically coupled quantum dots. Phys. Rev. B 75, 125317 (2007).

5. Imamoğlu, A. et al. Coupling quantum dot spins to a photonic crystal nanocavity. J. Appl. Phys. 101, 081602 (2007).

6. Jacac, L., Hawrylak, P. \& Wójs, A. Quantum Dots (Springer, Berlin, 1998).

7. Bimberg, D., Grundmann, M. \& Ledentsov, N. N. Quantum Dot Heterostructures (Wiley, New York, 1998).

8. Bayer, M., Stern, O., Hawrylak, P., Fafard, S. \& Forchel, A. Hidden symmetries in the energy levels of excitonic 'artificial atoms'. Nature 405, 923-926 (2000).

9. Drexler, H., Leonard, D., Hansen, W., Kotthaus, J. P. \& Petroff, P. M. Spectroscopy of quantum levels in charge-tunable InGaAs quantum dots. Phys. Rev. Lett. 73, 2252-2255 (1994).

10. Blokland, J. H. et al. Hole levels in InAs self-assembled quantum dots. Phys. Rev. B 75, 233305 (2007)

11. Narvaez, G. A. \& Zunger, A. Calculation of conduction-to-conduction and valence-to-valence transitions between bound states in (In,Ga)As/GaAs quantum dots. Phys. Rev. B 75, 085306 (2007).

12. Ediger, M. et al. Peculiar many-body effects revealed in the spectroscopy of highly charged quantum dots. Nature Phys. 3, 774-779 (2007).

13. Stinaff, E. A. et al. Optical signatures of coupled quantum dots. Science 311, 636-639 (2006).
14. Bracker, A. S. et al. Engineering electron and hole tunneling with asymmetric InAs quantum dot molecules. Appl. Phys. Lett. 89, 233110 (2006).

15. Scheibner, M. et al. Spin fine structure of optically excited quantum dot molecules. Phys. Rev. B 75, 245318 (2007).

16. Scheibner, M. et al. Photoluminescence spectroscopy of the molecular biexciton in vertically stacked InAs-GaAs quantum dot pairs. Phys. Rev. Lett. 99, 197402 (2007).

17. Krenner, H. J. et al. Direct observation of controlled coupling in an individual quantum dot molecule. Phys. Rev. Lett. 94, 057402 (2005).

18. Ortner, G. et al. Control of vertically coupled InGaAs/GaAs quantum dots with electric field. Phys. Rev. Lett. 94, 157401 (2005).

19. Krenner, H. J. et al. Optically probing spin and charge interactions in a tunable artificial molecule. Phys. Rev. Lett. 97, 076403 (2006).

20. Szafran, B., Peeters, F. M. \& Bednarek, S. Stark effect on the exciton spectra of vertically coupled quantum dots: Horizontal field orientation and nonaligned dots. Phys. Rev. B 75, 115303 (2007).

21. Degani, M. H. \& Maialle, M. Z. Resonances of trion states in quantum dot molecules tuned by an electric field. Phys. Rev. B 75, 115322 (2007).

22. Bester, G. \& Zunger, A. Electric field control and optical signature of entanglement in quantum dot molecules. Phys. Rev. B 72, 165334 (2005).

23. Beirne, G. J. et al. Quantum light emission of two lateral tunnel-coupled (In,Ga)As/GaAs quantum dots controlled by a tunable static electric field. Phys. Rev. Lett. 96, 137401 (2006).

24. Xie, Q., Madhukar, A., Chen, P. \& Kobayashi, N. P. Vertically self-organized InAs quantum box islands on GaAs(100). Phys. Rev. Lett. 75, 2542-2545 (1995).

25. Bruls, D. M. et al. Stacked low-growth-rate InAs quantum dots studied at the atomic level by cross-sectional scanning tunneling microscopy. Appl. Phys. Lett. 82, 3758-3760 (2003).

26. Solomon, G. S., Komarov, S., Harris, J. S. Jr \& Yamamoto, Y. Increased size uniformity through vertical quantum dot columns. J. Cryst. Growth 175-176, 707-712 (1997).

27. Jaskólski, W., Zielinski, M., Bryant, G. W. \& Aizpurua, J. Strain effects on the electronic structure of strongly coupled self-assembled InAs/GaAs quantum dots: Tight-binding approach. Phys. Rev. B 74, 195339 (2006).

28. Garcia, J. M. et al. Intermixing and shape changes during the formation of InAs self-assembled quantum dots. Appl. Phys. Lett. 71, 2014-2016 (1997).

29. Wasilewski, Z. R., Fafard, S. \& McCaffrey, J. P. Size and shape engineering of vertically stacked self-assembled quantum dots. J. Cryst. Growth 201-202, 1131-1135 (1999).

30. Nosho, B. Z., Barvosa-Carter, W., Yang, M. J., Bennett, B. R. \& Whitman, L. J. Interpreting interfacial structure in cross-sectional STM images of III-V semiconductor heterostructures. Surf. Sci. 465, $361-371$ (2000).

\section{Acknowledgements}

We acknowledge partial funding by NSA/ARO and ONR.

Correspondence and requests for materials should be addressed to M.S. or D.G.

Supplementary Information accompanies this paper on www.nature.com/naturephysics.

Reprints and permission information is available online at http://npg.nature.com/reprintsandpermissions/ 